Maximilian Neidhardt*, Stefan Gerlach*, Max-Heinrich Laves, Sarah Latus, Carolin Stapper, Martin Gromniak, and Alexander Schlaefer

\title{
Collaborative robot assisted smart needle placement
}

https://doi.org/10.1515/cdbme-2021-2120

\begin{abstract}
Needles are key tools to realize minimally invasive interventions. Physicians commonly rely on subjectively perceived insertion forces at the distal end of the needle when advancing the needle tip to the desired target.

However, detecting tissue transitions at the distal end of the needle is difficult since the sensed forces are dominated by shaft forces. Disentangling insertion forces has the potential to substantially improve needle placement accuracy. We propose a collaborative system for robotic needle insertion, relaying haptic information sensed directly at the needle tip to the physician by haptic feedback through a light weight robot. We integrate optical fibers into medical needles and use optical coherence tomography to image a moving surface at the tip of the needle. Using a convolutional neural network, we estimate forces acting on the needle tip from the optical coherence tomography data. We feed back forces estimated at the needle tip for real time haptic feedback and robot control. When inserting the needle at constant velocity, the force change estimated at the tip when penetrating tissue layers is up to $94 \%$ between deep tissue layers compared to the force change at the needle handle of $2.36 \%$. Collaborative needle insertion results in more sensible force change at tissue transitions with haptic feedback from the tip $(49.79 \pm 25.51) \%$ compared to the conventional shaft feedback $(15.17 \pm 15.92) \%$. Tissue transitions are more prominent when utilizing forces estimated at the needle tip compared to the forces at the needle shaft, indicating that a more informed advancement of the needle is possible with our system.
\end{abstract}

Keywords: robotics, biopsy, machine learning, haptic feedback

\section{Introduction}

Needle insertions are widely used in the clinical routine, e.g., in diagnosis (biopsies, blood sampling), therapy (brachytherapy, radiofrequency ablations), and anesthesia [1]. The most promi-

\footnotetext{
*Equal contribution

Maximilian Neidhardt, Stefan Gerlach, Max-Heinrich Laves, Sarah Latus, Carolin Stapper, Martin Gromniak, Alexander Schlaefer, Institute of Medical Technology and Intelligent Systems, Hamburg University of Technology, Hamburg, Germany, e-mail: \{firstname.lastname\}@tuhh.de
}

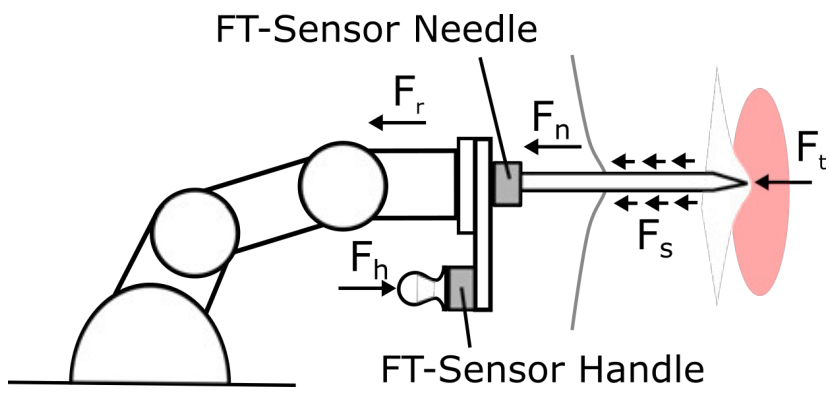

Fig. 1: Forces acting on the collaborative robotic system. While inserting the needle the surgeon applies a handle force $\left(F_{h}\right)$. Both the shaft force $\left(F_{f}\right)$ and the tip force $\left(F_{t}\right)$ act on the needle. We scale $F_{t}$ and feedback the force resulting in $F_{r}$. During insertion we measure the forces at the handle and the needle shaft.

nent method for needle guidance is ultrasound, however, it is limited by its penetration depth, shadowing by bones and soft tissue contrast. Without image guidance, the physician has to navigate the needle based only on the subjective sense of touch on the needle handle, which is subject to many limitations. The physician feels the sum of forces acting between needle tip and tissue and friction forces acting between the tissue and the shaft. We refer to these forces as tip forces and shaft forces, respectively (see Fig. 1). When a needle is inserted into soft tissue, the shaft forces increase continuously due to the increasing needle-tissue contact area [3]. In contrast, the tip force is directly related to the physiological properties of the tissue of interest in front of the needle tip. The tip force can therefore indicate a change of surrounding soft tissue, punctures of membranes [8] or boundaries of tumor tissue [2].

\section{Related Work}

In the past, we have developed needles that have integrated optical fibers for force sensing at the needle tip [6]. We employ optical coherence tomography (OCT) due to its high temporal sampling rate $(91 \mathrm{KHz})$ and high depth resolution $(\sim 10 \mu \mathrm{m})$ as imaging modality. With OCT, the deformation of an epoxy layer can be imaged, which is located behind a sharp cone tip at the tip of the needle (see Fig. 2 right). The image data is processed in real-time using machine learning methods. In preliminary experiments, we have already estimated forces during the insertion in human ex vivo tissue with a mean absolute error of $(1.59 \pm 1.30) \mathrm{mN}[4,5]$. Further, we have shown that 

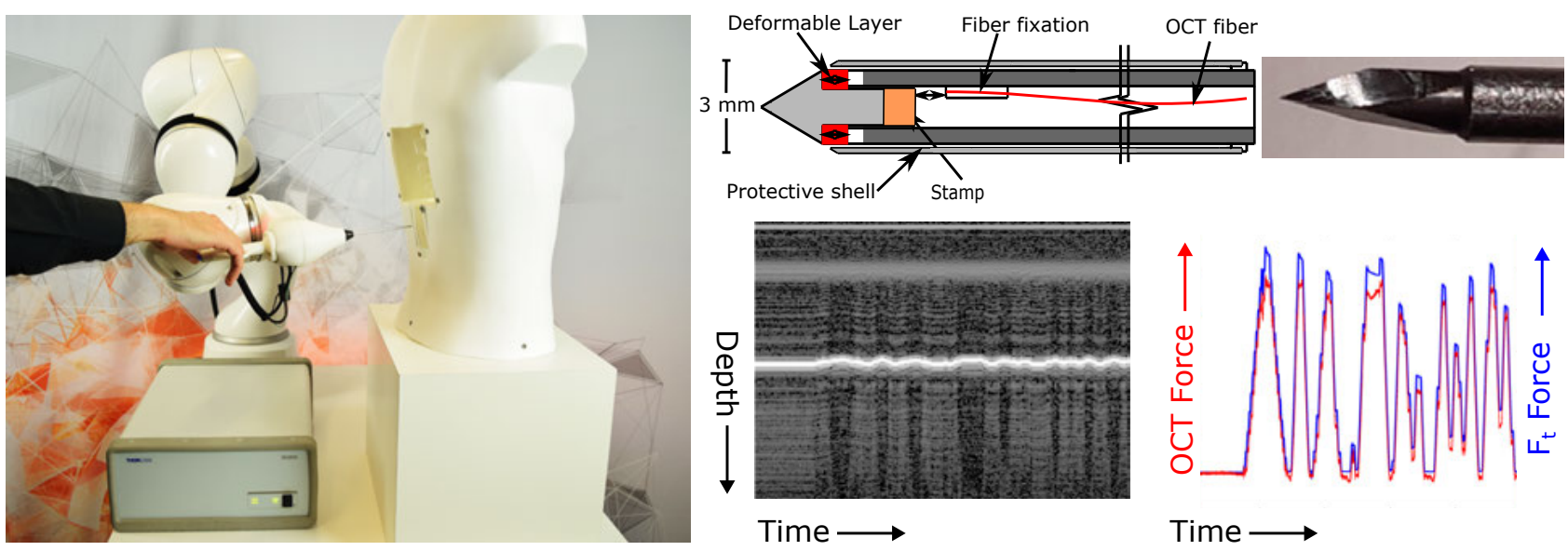

Fig. 2: The collaborative robotic needle insertion system. Depicted is the light weight robot, OCT system (bottom left) and a phantom to simulate needle placement during epidural anesthesia. First, the surgeon can position the needle relative to the insertion target by driving the robot using the handle. Second, with the push of a button at the top of the handle, the surgeon switches into the needle insertion mode. The robot only allows movements along the needle axis and the surgeon can feel the forces acting on the tip of the needle. Right Images: Schematic (topleft) and real (top-right) needle with an example of OCT image data (bottom-left) and predicted OCT force and measured force (bottom-right).

force estimation from raw OCT data outperforms the use of reconstructed OCT data [6]. In addition, we have placed medical biopsy needles with robots and sensed the forces acting in human corps tissue [7].

In this work, we extend the application of our smart force sensing needle and integrate it into a collaborative robot system for assisted needle insertion. The robot serves as a needle guidance and as a haptic feedback device. We estimate the tip force during an insertion in real-time with a convolutional neural network $(\mathrm{CNN})$. We feedback this information to the physician by applying a virtual force through the robotic arm.

Physicians commonly palpate the tissue with their bare fingers in open surgeries and use the perceived elasticity as a major criterion to distinguish between malignant and healthy tissue. Our collaborative robotic needle insertion system has the potential to enable the same for minimally invasive procedures, ultimately making the placement of the needle more informed and safer.

\section{Methods}

An overview of our collaborative needle insertion system is presented in Fig. 1. The system includes a 7-axis lightweight robot arm with a custom handle piece, two distally mounted force sensors, and a custom needle for proximal OCT-based force sensing.

\section{Robot and Handle}

We employ a KUKA LBR Med (KUKA AG, Augsburg, Germany) robot for needle placement. The physician is able to control the robot in two modes, which can be changed by the click of a button at the handle. First, the surgeon can position the needle in 6 degrees of freedom with one hand (Fig. 2). We measure the forces and torques acting on the handle and convert these into a translation and rotation of the needle, respectively. After a suitable position for inserting the needle is found, the surgeon can switch to the second mode. Now, the direction of movement is restricted to the axial needle direction and force feedback by the robot is enabled.

\section{Smart Needle}

Our smart needle $[4,6]$ is attached to the robot's end-effector and senses forces acting at the needle tip. The force at the needle tip results from the elastic stiffness of the tissue and the cutting force of the needle through the tissue. We integrate an OCT fiber to image deformation of a ductile layer due to forces acting at the needle tip (Fig. 2, top right). The deformable layer is produced from epoxy resin. The OCT fiber is fixed inside a ferule and aligned towards the movable stamp with a distance of approximately $1 \mathrm{~mm}$. The outer diameter of our needle is $3 \mathrm{~mm}$. For imaging, we employ a Thorlabs OCT device (ThorLabs, Inc., Newton, NJ, USA) with a temporal resolution of $91 \mathrm{kHz}$. We have shown a mean absolute error of $5.81 \mathrm{mN}$ when calibrated for forces between $0 \mathrm{~N}$ and $1 \mathrm{~N}$.

We use a $\mathrm{CNN}$ to process the image data and predict forces acting on the needle tip. To allow for fast training and real-time inference, we adapt and reduce a CNN we previously presented [5]. The training data for a needle attached to the robot is 

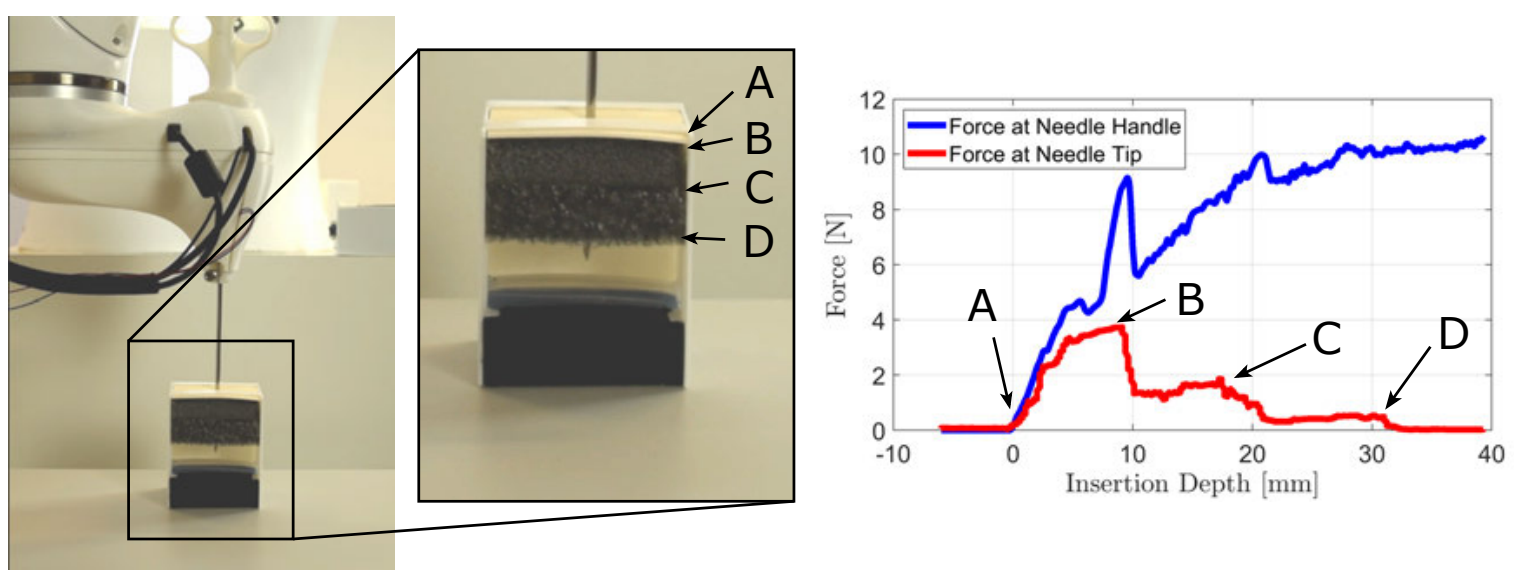

Fig. 3: Needle insertion into a multi-layered phantom (left) with corresponding forces (right). The robot drives with a constant velocity. At $\mathbf{A}$ the needle makes contact with the skin. At $\mathbf{B}$ the needle has passed the skin. At $\mathbf{C}$ the needle passes from the first to the second layer. At $\mathbf{D}$ the needle passes the second softer layer.
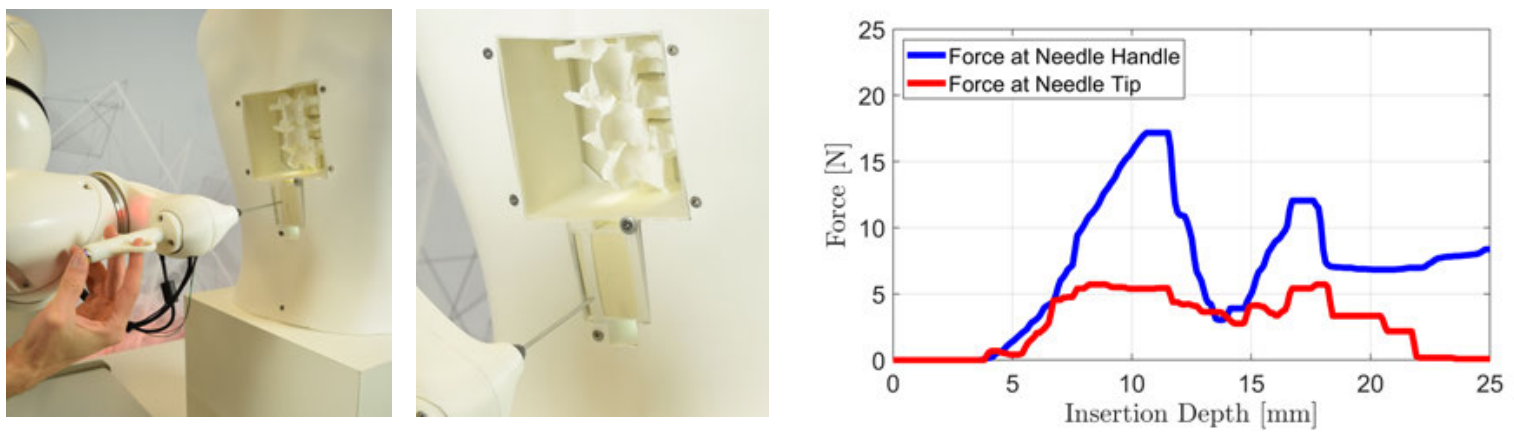

Fig. 4: Collaborative needle insertion into a single-layered phantom. The robot only allows movement along the needle shaft in needle insertion mode. The mode can be changed with the push of the button at the top of the handle (left). The phantom is mounted inside a thorso (center). The needle has to penetrate a skin layer followed by a styrofoam layer. The forces at the needle handle vary strongly during the insertion compared to the forces estimated at the tip. After passing the styrofoam layer the user will not feel any haptic feedback from the robot since no forces are sensed at the tip compared to conventional needle placement with forces sensed at the handle.

created by repeatedly driving it by hand on a solid surface with up to $10 \mathrm{~N}$. We measure the forces at the needle shaft and use this information as training labels (Fig. 1, bottom right). The data generation procedure is performed for 2 minutes. The complete data generation and training procedure is completed in 6-10 minutes.

\section{Control Loop}

We design a feedback loop to control the pose of the robot by targeting an equilibrium of the force predicted at the needle tip $\left(F_{t}\right)$ and the force exerted at the needle handle $\left(F_{h}\right)$ by the physician. We implement admittance control with a PID controller to minimize the error $E_{f}$, where

$$
E_{f}=F_{h}+s \cdot F_{t},
$$

with $s$ as a linear scaling factor to increase the haptic feedback. The PID controller updates the pose of the robot during insertions with a loop frequency of $1000 \mathrm{~Hz}$.

\section{Results}

We evaluate our system by driving into multi-layered phantoms resembling haptic properties of tissue. The forces acting on the handle and tip of the needle during an insertion with constant velocity are shown in Fig. 3. The transitions between the three layers are clearly visible in the change of tip force of $64 \%$, $72 \%$, and $94 \%$ between tissue layers. The forces acting on the needle handle are quickly dominated by friction force. Here, the force changes by $38 \%, 9 \%$, and $2 \%$ between layers. Only the first phantom layers can be clearly distinguished. The tip force considerably decreases for softer layers with increased penetration depth.

The forces acting on the handle and tip of the needle during an insertion with haptic feedback through the robot from the estimated needle tip force is shown in Fig. 4. The force at the needle handle varies distinctly more than at the tip due to 
velocity changes controlled by the user. After passing through the styrofoam layer, no tip force can be measured since the needle tip is in air, in contrast to the still significant handle force. Overall, the relative force change at tissue transitions with haptic feedback from the tip is considerably larger with $(49.79 \pm 25.51) \%$ compared to the conventional shaft feedback with $(15.17 \pm 15.92) \%$.

\section{Discussion}

We have established a collaborative robotic needle insertion system with robotic haptic feedback. In conjunction with force sensing at the tip of a needle, we can make transitions between tissue layers palpable. Considering Fig. 3, we show that the force, which the physician feels while inserting the needle with our system, can help identify tissue layer transitions. Further, we can identify the elasticity of distinct layers. The force felt by the physician during a conventional needle insertion (needle handle force) increases as the needle is advanced, making it difficult to detect tissue transitions and elastic properties of the tissue.

Furthermore, first results with a user operating the system indicate that transitions between tissue layers can be felt more clearly with feedback from the needle tip force in comparison to feedback from conventional shaft force. Hence, a more informed decision can be made when advancing a needle without line of sight.

Additionally our system does not rely on offline planned robotic trajectories. Using tip force feedback, the physician can detect tissue deformation online and can place the needle more accurately. Still, recording data of needle insertions into real tissue with our collaborative system is crucial on the way towards fully autonomous needle insertions. With the proposed system, clinical data of needle insertions can be recorded by experienced physicians, as the surgeon has control over the robot and thus the needle position in the patient at all times. Furthermore, clinical evaluation of the impact of the increased haptic information from the needle tip is necessary for estimation of medical applicability.

\section{Conclusion}

We have integrated a collaborative robot assisted system for needle placement. During needle insertion we sense forces at the needle tip and apply real-time force feedback through the robot to the surgeon. The system has the potential to improve needle placement accuracy through haptic sensing of tissue needle interaction at the tip of the needle. This allows the surgeon to feel the tissue without a line of sight and make a more informed decision during the advancement of the needle.

\section{Author Statement}

Research funding: The robot used in this study was provided by KUKA as part of the KUKA Innovation Award 2020. This work was partially funded by DFG SCHL 1844/2-2.

Conflict of interest: Authors state no conflict of interest. Informed consent: Informed consent has been obtained from all individuals included in this study. Ethical approval: The research related to human use complies with all the relevant national regulations, institutional policies and was performed in accordance with the tenets of the Helsinki Declaration, and has been approved by the authors' institutional review board or equivalent committee.

\section{References}

[1] N. Abolhassani, R. Patel, and M. Moallem. Needle insertion into soft tissue: A survey. Med Eng Phys, 29(4):413-431, 2007.

[2] D. Chauvet, M. Imbault, L. Capelle, C. Demene, M. Mossad, C. Karachi, A. Boch, J. Gennisson, and M. Tanter. In vivo measurement of brain tumor elasticity using intraoperative shear wave elastography. Ultraschall Med, 37(6):584-590, 2016.

[3] S. P. DiMaio and S. E. Salcudean. Needle insertion modeling and simulation. IEEE Trans Robot Autom, 19(5):864-875, 2003.

[4] N. Gessert, T. Priegnitz, T. Saathoff, S.-T. Antoni, D. Meyer, M. F. Hamann, K.-P. Jünemann, C. Otte, and A. Schlaefer. Needle tip force estimation using an OCT fiber and a fused convgru-cnn architecture. In Int J Comput Assist Radiol Surg, pages 222-229. Springer, 2018.

[5] N. Gessert, T. Priegnitz, T. Saathoff, S.-T. Antoni, D. Meyer, M. F. Hamann, K.-P. Jünemann, C. Otte, and A. Schlaefer. Spatiotemporal deep learning models for tip force estimation during needle insertion. Int J Comput Assist Radiol Surg, 14(9):14851493, 2019.

[6] M. Gromniak, N. Gessert, T. Saathoff, and A. Schlaefer. Needle tip force estimation by deep learning from raw spectral OCT data. Int J Comput Assist Radiol Surg, 15(10):1699-1702, 2020.

[7] M. Gromniak, M. Neidhardt, A. Heinemann, K. Püschel, and A. Schlaefer. Needle placement accuracy in CT-guided robotic post mortem biopsy. Curr Dir Biomed Eng, 6(1), 2020.

[8] G. Tholey, J. P. Desai, and A. E. Castellanos. Force feedback plays a significant role in minimally invasive surgery: results and analysis. Ann Surg, 241(1):102, 2005. 